\title{
Suscetibilidade de Cultivares de ARRoz IRRigado (Oryza sativa) À DERIVA SIMULADA DO HERBICIDA IMAZETHAPYR + IMAZAPIC ${ }^{1}$
}

\author{
Susceptibility of Rice Cultivars (Oryza sativa) to Simulated Drift of the Herbicide \\ Imazethapyr + Imazapic
}

\author{
DAL MAGRO, T. ${ }^{2}$, PINTO, J.J.O. ${ }^{3}$, AGOSTINETTO, D. ${ }^{3}$, GALON, L. ${ }^{4}$, REZENDE, A.L. ${ }^{4}$ e \\ LAZAROTTO, C.A. ${ }^{4}$
}

\begin{abstract}
RESUMO - Objetivou-se neste trabalho avaliar a suscetibilidade dos cultivares de arroz irrigado IRGA 417, BR-IRGA 410, BRS Pelota e Gualimax 1 à simulação de deriva do herbicida imazethapyr + imazapic em função da época de início da irrigação por inundação. Para isso, foi conduzido experimento em casa de vegetação do Departamento de Fitossanidade da FAEM/ UFPel, na estação de crescimento 2003/04. O delineamento experimental utilizado foi o de blocos casualizados, com quatro repetições. A deriva foi simulada pela aplicação de doses crescentes do herbicida imazethapyr + imazapic, correspondendo a $0 ; 3,125 ; 6,25 ; 12,5 ; 25$; 50; e 100\% da dose comercial (100 $\mathrm{g} \mathrm{ha}^{-1}$ ), acrescido de Dash a 0,5\% v/v, pulverizados sobre as plantas de arroz em estádios fenológicos V3 - V4, com inundação três dias antes ou após a aplicação dos tratamentos. A aplicação foi realizada com pulverizador costal, pressurizado a $\mathrm{CO}_{2}$, munido de barra com bicos 110.015 do tipo leque a uma pressão constante de $210 \mathrm{kPa}$, que proporcionou a aplicação de $150 \mathrm{~L} \mathrm{ha}^{-1}$ de calda herbicida. A fitotoxicidade ocasionada pelo herbicida foi avaliada de forma visual aos 10, 15 e 20 dias após a aplicação dos tratamentos (DAT), utilizando-se escala percentual. Aos 25 DAT foi determinada a massa seca aérea e radical. O herbicida imazethapyr + imazapic causou fitotoxicidade aos cultivares de arroz IRGA 417, BR-IRGA 410, BRS Pelota e Qualimax 1, em deriva ocorrida em solo seco ou inundado. A massa seca da parte aérea e radical foi reduzida com o aumento da dose aplicada, independentemente da condição de aplicação. O cultivar IRGA 417 foi mais sensivel à simulação de deriva de imazethapyr + imazapic.
\end{abstract}

Palavras-chave: fitotoxicidade, massa seca, imazethapyr + imazapic.

\begin{abstract}
The susceptibility of rice cultivars IRGA 417, BR-IRGA 410, BRS Pelota and Qualimax 1 was evaluated using a simulated drift of the herbicide imazethapyr + imazapic as a function of timing of flooding. The experiment was conducted under greenhouse conditions during the $2003 / 2004$ growing season. The experimental design was arranged in randomized blocks with four replications. Treatments consisted of drift simulation by applying increasing herbicide doses at the following rates: $0 \%, 3.125 \% ; 6.25 \% ; 12.5 \% ; 25 \%$; $50 \%$ or $100 \%$ of the commercial dose (amended with Dash at $0.5 \% \mathrm{v} / \mathrm{v}$ ), on rice plants at the V3 to V4 growth stages. Flooding was applied either three days prior or after treatment application. Herbicide was applied using a $\mathrm{CO}_{2}$ pressurized costal sprayer (flat fan nozzles 110.015 on the boom) at $150 \mathrm{~L} \mathrm{ha}^{-1}$ with a constant pressure of $210 \mathrm{kPa}$. Phytotoxicity, expressed in percentage, was evaluated visually at 10, 15 and 20 days after treatment (DAT). Plant and root dry mass were evaluated at 25 DAT. Results showed that herbicide drift promoted toxic effects to rice plant under both dry and flooding conditions. Plant dry mass was significantly reduced with increasing herbicide dosage, regardless of timing of flooding. Cultivar IRGA 417 was the most sensitive to imazethapyr + imazapic simulated drift.
\end{abstract}

Keywords: phytotoxicity, dry mass, imazethapyr + imazapic.

Recebido para publicação em 12.4.2006 e na forma revisada em 10.11.2006.

Eng ${ }^{\mathrm{a}}$--Agr ${ }^{\mathrm{a}}$., aluna do Programa de Pós-Graduação em Fitossanidade da Universidade Federal de Pelotas - UFPel, Caixa Postal 354, 96010-900 Capão do Leão-RS, <taisadm@yahoo.com.br >; ${ }^{3}$ Professor da Faculdade de Agronomia Eliseu Maciel UFPel. ${ }^{4}$ Aluno de graduação da Faculdade de Agronomia Eliseu Maciel - UFPel. 


\section{INTRODUÇÃO}

O arroz-vermelho (Oryza sativa) é uma das mais importantes plantas daninhas em áreas produtoras de arroz do mundo (Eleftherohorinos \& Dhima, 2002). A sua presença em lavoura comercial, além de reduzir a produtividade, diminui o custo de produção, decorrente do acréscimo de práticas adicionais de controle (Noldin et al., 2004). O controle do arroz-vermelho em lavouras infestadas deve ser feito com a utilização de um conjunto integrado de práticas, que incluem o uso de sementes puras, sistema de semeadura com sementes pré-germinadas, transplantio, preparo do solo na entressafra, adoção do sistema de cultivo mínimo (Noldin et al., 2004), práticas de rotação de culturas e controle químico (Ferrero et al., 1999). No entanto, o método químico com herbicidas seletivos torna-se inviável devido às similaridades botânicas entre o arroz cultivado e o arroz-vermelho (Eleftherohorinos \& Dhima, 2002). A mais recente tecnologia para controle seletivo de arroz-vermelho foi o desenvolvimento de cultivares tolerantes a herbicidas do grupo das imidazolinonas (Ottis et al., 2003). Esse sistema denomina-se Clearfield ${ }^{\square}$ e, no Brasil, o herbicida registrado é o Only ${ }^{\square}$, o qual possui 75 e 25 g de imazethapyr e imazapic, respectivamente.

Dente os fatores que afetam a sensibilidade da cultura do arroz aos herbicidas, destacamse o cultivar utilizado, o herbicida e/ou dose aplicada e a época de início da irrigação permanente. Com relação a cultivares, é possivel observar resposta diferenciada entre herbicidas e doses do produto. Estudos realizados por Pinto et al. (2003) com o herbicida bispiribacsodium demonstraram diferenças de sensibilidade entre os cultivares BRS Pelota e EPAGRI 112. Resultados obtidos por Benitez (1997) indicam que a aplicação dos herbicidas quinclorac e propanil ocasionou sintomas de fitotoxicidade às plantas dos cultivares BR IRGA 414, EMBRAPA 6-Chuí e EMBRAPA 7Taim. Esses sintomas reduziram com o passar do tempo, observando-se recuperação total das plantas aos 21 dias após a aplicação. Nesse mesmo estudo, foi detectado que o herbicida clomazone ocasionou maiores índices de injúria às plantas de arroz; o cultivar mais sensivel foi o EMBRAPA 7-Taim.
Outro fator que influencia a fitotoxicidade dos herbicidas às plantas é a época de início da irrigação por inundação, visto que a presença da lâmina de água nas lavouras altera as condições de estabelecimento e desenvolvimento da cultura e das plantas daninhas.

Os herbicidas, em sua grande maioria, podem causar danos ao meio ambiente, razão pela qual a sua utilização requer cuidados especiais quando da ocorrência de condições favoráveis de deriva. A deriva é considerada o movimento do produto no ar, durante ou após a aplicação, para um local diferente daquele para o qual está sendo planejado. A parte do produto pulverizado que não atinge o alvo esperado pode ocasionar efeitos deletérios ao aplicador, a culturas sensiveis, animais e mananciais hídricos. A deriva é influenciada por diversos fatores, dentre os quais podem-se destacar o equipamento e as técnicas de aplicação, as condições climáticas e a habilidade do operador na aplicação do herbicida. Devese sempre ressaltar que a utilização de herbicidas não-seletivos requer maior cuidado no momento da aplicação.

Estudos com deriva foram realizados com glyphosate e butroxydim nos cultivares Qualimax 1 e EMBRAPA 6-Chuí, respectivamente (Ferreira, 2003); com glyphosate, nos cultivares Lemont e Priscila (Kurtz \& Street, 2003); e com glyphosate e glufosinate, no cultivar Cypress (Ellis et al., 2003). De modo geral, os resultados apontam que a deriva causou injúrias crescentes com a dose, reduzindo a produtividade da cultura.

A hipótese da pesquisa foi de que o controle químico de plantas daninhas em lavouras cultivadas com arroz mutante poderá causar deriva a lavouras de arroz sensível e que a injúria poderá variar em função do cultivar e com a época de início da irrigação definitiva.

O objetivo deste trabalho foi avaliar a injúria causada pela simulação de deriva de herbicidas do grupo das imidazolinonas aos cultivares de arroz IRGA 417, BR-IRGA 410, BRS Pelota e Qualimax 1, em decorrência do início da irrigação por inundação.

\section{MATERIAL E MÉTODOS}

Um experimento foi realizado em condições de casa de vegetação, no Departamento de 
Fitossanidade da Faculdade de Agronomia Eliseu Maciel da Universidade Federal de Pelotas (UFPel), município de Capão do Leão-RS, durante a estação de crescimento 2003/04. O solo utilizado é classificado como Planossolo Hidromórfico Eutrófico Solódico, pertencente à Unidade de Mapeamento Pelotas (EMBRAPA, 1999).

O delineamento experimental foi o de blocos ao acaso, com quatro repetições. As unidades experimentais constaram de baldes plásticos com capacidade para oito litros. Os cultivares foram IRGA 417, de ciclo precoce, BR-IRGA 410, BRS Pelota e Gualimax 1, de ciclo médio (SOSBAI, 2003). O arroz foi semeado manualmente, utilizando-se 10 sementes por balde; após a emergência das plantas foi realizado desbaste, corrigindo-se a população para sete plantas por balde, densidade esta correspondente à população de 170 plantas $\mathrm{m}^{-2}$.

A deriva do herbicida foi simulada pela aplicação de doses crescentes de imazethapyr + imazapic, correspondendo a 0; 3,125; 6,$25 ; 12,5 ; 25 ; 50$; e $100 \%$ da dose comercial (100 $\mathrm{g} \mathrm{ha}^{-1}$ ), acrescido de Dash a 0,5\% v/v, pulverizados sobre as plantas de arroz em estádios fenológicos V3-V4 (SOSBAI, 2003). Três dias antes ou após a aplicação dos tratamentos, os vasos foram inundados; neste trabalho, serão mencionados como mantidos inundados ou no seco, respectivamente.

A aplicação do herbicida foi feita com pulverizador costal, pressurizado a $\mathrm{CO}_{2}$, munido com bico 110.015 do tipo leque a uma pressão constante de $210 \mathrm{kPa}$, proporcionando a aplicação de $150 \mathrm{~L} \mathrm{ha}^{-1}$ de calda herbicida.

A fitotoxicidade do herbicida foi quantificada por avaliações realizadas visualmente, aos 10, 15 e 20 dias após a aplicação dos tratamentos (DAT), utilizando-se escala de 0 a $100 \%$, em que 0 e 100 corresponderam à ausência de injúria e morte total das plantas, respectivamente.

A massa seca da parte aérea e radical foi determinada aos 25 DAT, pela coleta e secagem em estufa a $65{ }^{\circ} \mathrm{C}$, até peso constante das plantas contidas em cada unidade experimental (baldes).

Os dados foram analisados quanto à sua homocedasticidade, sendo posteriormente submetidos à análise de variância $(\mathrm{Pd0}, 05)$. Os efeitos de época de irrigação foram avaliados pelo teste $\mathrm{t}(\mathrm{PdO}, 05)$; os de cultivar, pelo teste de Duncan (Pd0,05) (Zonta et al., 1984); e os do fator dose, por modelos de regressão nãolinear $(\mathrm{PdO}, 05)$ (Sigmaplot, 1999), conforme segue:

$$
\begin{aligned}
& Y=a e^{-b x} \\
& Y=a\left(1-e^{-b x}\right)
\end{aligned}
$$

em que $a=$ valor máximo estimado para a variável resposta; $b=$ inclinação da curva; $x=$ dose do herbicida imazethapyr + imazapic (g ha-1); $e=$ constante.

\section{RESULTADOS E DISCUSSÃO}

A fitotoxicidade observada para o cultivar IRGA 417 na primeira avaliação, 10 dias após a aplicação dos tratamentos (DAT), apresentou diferença em função das condições de aplicação para doses até $25 \mathrm{~g}_{\text {ha }}{ }^{-1}$ (Tabela 1). Na segunda (15 DAT) e na terceira (20 DAT) avaliação foi observada diferença apenas na menor dose testada.

Com relação ao cultivar de arroz BR-IRGA 410 , a fitotoxicidade verificada na primeira avaliação diferiu entre as duas condições de aplicação, nas doses de 3,$125 ; 6,25$; 50; e $100 \mathrm{~g} \mathrm{ha}^{-1}$ (Tabela 1). Contudo, para a segunda avaliação, as doses que diferiram foram de 3,125 e $12,5 \mathrm{~g} \mathrm{ha}^{-1}$. Na última avaliação não foi observada diferença entre as duas condições de aplicação em nenhuma das doses testadas.

Para o cultivar BRS Pelota, na primeira avaliação, diferenças foram observadas nas doses maiores que $3,125 \mathrm{~g} \mathrm{ha}^{-1}$ (Tabela 1). Entretanto, na segunda e terceira avaliações, não foram observadas diferenças entre as doses testadas em função das duas condições de aplicação.

Para o cultivar Qualimax 1, na primeira avaliação, diferenças em função das condições de ocorrência da deriva foram constatadas em todas as doses, à exceção da de $3,125 \mathrm{~g} \mathrm{ha}^{-1}$ (Tabela 1). Na segunda avaliação, as doses que apresentaram diferenças em função da condição da aplicação foram 6,25 e 12,5 $\mathrm{g} \mathrm{ha}^{-1}$. Já na última avaliação, apenas as doses de 25 e $50 \mathrm{~g} \mathrm{ha}^{-1}$ diferiram de acordo com as condições de aplicação. 
Tabela 1 - Fitotoxicidade causada por deriva simulada de imazethapyr + imazapic a cultivares de arroz irrigado, em função de dose e condição da aplicação, em três épocas de avaliação. CAP/UFPel, Capão do Leão-RS, 2003/04

\begin{tabular}{|c|c|c|c|c|c|c|c|c|c|c|c|c|c|c|c|c|}
\hline \multirow{2}{*}{$\begin{array}{l}\text { Dose } \\
\left(\mathrm{g} \mathrm{ha}^{-1}\right)^{1^{\prime}}\end{array}$} & \multicolumn{4}{|c|}{ IRGA 417} & \multicolumn{4}{|c|}{ BR-IRGA 410} & \multicolumn{4}{|c|}{ BRS Pelota } & \multicolumn{4}{|c|}{ Qualimax 1} \\
\hline & \multicolumn{2}{|l|}{ Seco } & \multicolumn{2}{|l|}{ Inundado } & \multicolumn{2}{|l|}{ Seco } & \multicolumn{2}{|l|}{ Inundado } & \multicolumn{2}{|l|}{ Seco } & \multicolumn{2}{|l|}{ Inundado } & \multicolumn{2}{|l|}{ Seco } & \multicolumn{2}{|l|}{ Inundado } \\
\hline & \multicolumn{16}{|c|}{$10 \mathrm{DAT}^{2 /}$} \\
\hline 0,00 & $0,00^{\mathrm{ns}}$ & $a^{\frac{3}{1}}$ & $0,00 \quad \mathrm{a}$ & & $0,00^{\mathrm{ns}} \mathrm{a}$ & $\mathrm{a}$ & 0,00 & $\mathrm{a}$ & $0,00^{\text {ns }}$ & $\mathrm{a}$ & 0,00 & $\mathrm{a}$ & $0,00^{\mathrm{ns}}$ & $\mathrm{a}$ & 0,00 & $\mathrm{a}$ \\
\hline 3,125 & $60,45 *$ & $\mathrm{a}$ & $40,00 \quad a$ & & $40,71 *$ & & 0,00 & $\mathrm{~b}$ & $0,00^{\text {ns }}$ & c & 0,00 & $\mathrm{~b}$ & $0,00^{\mathrm{ns}}$ & c & 0,00 & b \\
\hline 6,25 & $69,86 *$ & $\mathrm{a}$ & $60,00 \quad a$ & $\mathrm{a}$ & $59,96 *$ & $\mathrm{~b}$ & 48,67 & b & $70,22 *$ & a & 49,71 & $\mathrm{~b}$ & 70,00 * & $\mathrm{a}$ & 45,00 & $\mathrm{c}$ \\
\hline 12,50 & $69,96 *$ & $\mathrm{a}$ & 60,00 & $\mathrm{a}$ & $61,23^{\text {ns }}$ & & 59,19 & a & $69,96 *$ & & 60,46 & $\mathrm{a}$ & $70,00 *$ & $\mathrm{a}$ & 60,00 & $\mathrm{a}$ \\
\hline 25,00 & $74,96 *$ & b & 70,00 & $\mathrm{a}$ & $68,23^{\text {ns }}$ & c & 68,70 & a & $80,00 *$ & & 69,96 & a & $75,00 *$ & b & 70,00 & \\
\hline 50,00 & $79,96^{\mathrm{ns}}$ & $\mathrm{b}$ & 80,00 a & $\mathrm{a}$ & $71,23 *$ & c & 74,96 & $\mathrm{~b}$ & $84,96 *$ & a & 69,96 & c & $80,00 *$ & b & 70,00 & c \\
\hline \multirow[t]{2}{*}{100,00} & $89,74^{\mathrm{ns}}$ & $\mathrm{a}$ & $90,00 \quad \mathrm{a}$ & $\mathrm{a}$ & $84,96 *$ & $\mathrm{~b}$ & 79,96 & $\mathrm{~b}$ & $89,97 *$ & a & 79,72 & $\mathrm{~b}$ & $85,00 *$ & $\mathrm{~b}$ & 80,00 & $\mathrm{~b}$ \\
\hline & \multicolumn{16}{|c|}{$15 \mathrm{DAT}$} \\
\hline 0,00 & $0,00^{\mathrm{ns}}$ & $\mathrm{a}$ & $0,00 \quad \mathrm{a}$ & & $0,00^{\mathrm{ns}} \mathrm{a}$ & & 0,00 & $\mathrm{a}$ & $0,00^{\mathrm{ns}}$ & & 0,00 & & $0,00^{\mathrm{ns}}$ & $\mathrm{a}$ & 0,00 & \\
\hline 3,125 & $62,43 *$ & $\mathrm{a}$ & 54,89 & & $35,88 * \mathrm{~b}$ & & 0,00 & b & $0,00^{\text {ns }}$ & $\mathrm{c}$ & 0,00 & $\mathrm{~b}$ & $0,00^{\mathrm{ns}}$ & c & 0,00 & b \\
\hline 6,25 & $83,74^{\mathrm{ns}}$ & $\mathrm{a}$ & 79,99 a & $\mathrm{a}$ & $77,48^{\mathrm{ns}}$ & $\mathrm{b}$ & 78,58 & a & $79,46^{\mathrm{ns}}$ & $\mathrm{ab}$ & 78,73 & $\mathrm{a}$ & $80,00 *$ & $\mathrm{ab}$ & 70,00 & $\mathrm{~b}$ \\
\hline 12,50 & $79,96^{\text {ns }}$ & $\mathrm{a}$ & $80,46 \quad a$ & $\mathrm{a}$ & $81,24 *$ & a & 75,00 & b & $84,48^{\text {ns }}$ & a & 84,23 & $\mathrm{a}$ & 84,96 * & $\mathrm{a}$ & 70,00 & \\
\hline 25,00 & $79,99^{\text {ns }}$ & $\mathrm{b}$ & 84,96 a & & $84,25^{\mathrm{ns}}$ & $\mathrm{ab}$ & 81,74 & $\mathrm{~b}$ & $89,46^{\mathrm{ns}}$ & a & 89,40 & $\mathrm{a}$ & $80,00^{\mathrm{ns}}$ & $\mathrm{b}$ & 76,19 & \\
\hline 50,00 & $94,22^{\mathrm{ns}}$ & $\mathrm{a}$ & 93,74 a & $\mathrm{a}$ & $88,74^{\mathrm{ns}}$ & $a$ & 83,74 & $\mathrm{~b}$ & $90,00^{\mathrm{ns}}$ & a & 92,45 & $\mathrm{a}$ & $90,00^{\mathrm{ns}}$ & $\mathrm{a}$ & 86,24 & b \\
\hline \multirow[t]{2}{*}{100,00} & $96,99^{\text {ns }}$ & $\mathrm{a}$ & $95,71 \quad a$ & a & $94,23^{\mathrm{ns}}$ & $\mathrm{a}$ & 92,48 & a & $96,98^{\text {ns }}$ & a & 96,74 & $\mathrm{a}$ & $95,47^{\text {ns }}$ & $\mathrm{a}$ & 96,74 & a \\
\hline & \multicolumn{16}{|c|}{20 DAT } \\
\hline 0,00 & $0,00^{\text {ns }}$ & $\mathrm{a}$ & $0,00 \quad \mathrm{a}$ & & $0,00^{\mathrm{ns}} \mathrm{a}$ & $\mathrm{a}$ & 0,00 & $\mathrm{a}$ & $0,00^{\text {ns }}$ & & 0,00 & $\mathrm{a}$ & $0,00^{\mathrm{ns}}$ & $\mathrm{a}$ & 0,00 & \\
\hline 3,125 & $51,17 *$ & $\mathrm{a}$ & $29,90 \mathrm{a}$ & & $15,88^{\mathrm{ns}}$ & $\mathrm{b}$ & 16,20 & $\mathrm{~b}$ & $0,00^{\mathrm{ns}}$ & c & 0,00 & $\mathrm{c}$ & $0,00^{\mathrm{ns}}$ & $\mathrm{c}$ & 0,00 & \\
\hline 6,25 & $84,96^{\text {ns }}$ & $\mathrm{a}$ & $80,46 \quad a$ & & $77,97^{\text {ns }}$ & $\mathrm{b}$ & 81,74 & a & $84,96^{\mathrm{ns}}$ & & 80,23 & & $85,00^{\mathrm{ns}}$ & a & 80,00 & \\
\hline 12,50 & $84,96^{\mathrm{ns}}$ & $\mathrm{a}$ & $79,96 \quad a$ & & $80,73^{\text {ns }}$ & & 84,96 & a & $84,99^{\text {ns }}$ & & 79,99 & $\mathrm{a}$ & $85,00^{\mathrm{ns}}$ & $\mathrm{a}$ & 80,00 & \\
\hline 25,00 & $84,96^{\text {ns }}$ & $\mathrm{b}$ & $80,46 \quad b$ & $\mathrm{~b}$ & $88,74^{\mathrm{ns}}$ & $a b$ & 89,97 & a & $89,97^{\mathrm{ns}}$ & $a b$ & 84,96 & & $92,45 *$ & a & 85,00 & \\
\hline 50,00 & $89,97^{\text {ns }}$ & $\mathrm{b}$ & $90,49 \quad$ & & $95,48^{\text {ns }}$ & $a b$ & 91,73 & a & $95,24^{\text {ns }}$ & $a b$ & 95,00 & $\mathrm{a}$ & $97,74 *$ & $\mathrm{a}$ & 90,00 & \\
\hline 100,00 & $97,99^{\text {ns }}$ & $\mathrm{a}$ & $99,50 \quad \mathrm{a}$ & $\mathrm{a}$ & $99,25^{\text {ns }}$ & $\mathrm{a}$ & 97,24 & a & $95,99^{\text {ns }}$ & $\mathrm{a}$ & 97,24 & $\mathrm{a}$ & $99,50^{\mathrm{ns}}$ & $\mathrm{a}$ & 99,50 & \\
\hline
\end{tabular}

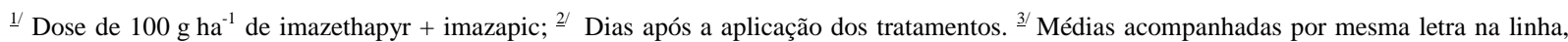
comparando cultivares, em cada condição de aplicação, não diferem entre si pelo teste de Duncan a 5\% de probabilidade.

${ }^{n s}$ e * Não-significativo e significativo, respectivamente, pelo teste t a 5\% de probabilidade em função da condição de aplicação.

No que se refere à fitotoxicidade causada aos cultivares, pode-se observar na primeira avaliação que o cultivar IRGA 417 apresentou fitotoxicidade média 14,9 e $21,7 \%$ superior à dos demais cultivares em solo seco e inundado, respectivamente (Tabela 1). Resultados semelhantes foram observados na segunda e terceira avaliações.

Segundo Benitez (1997), pode ocorrer diferença na tolerância de cultivares de arroz irrigado, que pode variar em função da dose e do herbicida. Esse autor verificou que para o herbicida clomazone os cultivares mais sensiveis foram EMBRAPA 7-Taim, EMBRAPA 6-Chuí e BR IRGA 414. Já para o herbicida bispiribac-sodium, o cultivar mais sensível foi EPAGRI 112, e o de menor sensibilidade, BRS Pelota (Pinto et al., 2003).
Para a variável massa seca da parte aérea, foi verificada diferença entre as duas condições de aplicação apenas para o cultivar IRGA 417, na menor dose testada (Tabela 2). Nos demais tratamentos, independentemente da dose ou do cultivar, não ocorreu diferença.

Maiores reduções de massa seca aérea foram constatadas no cultivar IRGA 417, sendo em média 46,9 e 16,2\% inferiores à média dos demais cultivares, para aplicação em solo seco e inundado, respectivamente (Tabela 2).

Com relação à produção de massa seca radical, os resultados se assemelharam aos de massa seca da parte aérea, sendo a produção observada para o cultivar IRGA 417 em média 78 e 39,6\% inferior à média dos demais cultivares nas duas condições de aplicação, respectivamente (Tabela 2). 
Tabela 2 - Efeito de deriva simulada de imazethapyr + imazapic na massa seca da parte aérea e radical de cultivares de arroz irrigado, em função de dose e condição da aplicação. CAP/UFPel, Capão do Leão-RS, 2003/04

\begin{tabular}{|c|c|c|c|c|c|c|c|c|c|c|c|c|c|c|c|}
\hline \multirow{2}{*}{$\begin{array}{l}\text { Dose } \\
\left(\mathrm{g} \mathrm{ha}^{-1}\right)^{\frac{1}{}}\end{array}$} & \multicolumn{4}{|c|}{ IRGA 417} & \multicolumn{4}{|c|}{ BR-IRGA 410} & \multicolumn{4}{|c|}{ BRS Pelota } & \multicolumn{3}{|c|}{ Qualimax 1} \\
\hline & \multicolumn{2}{|l|}{ Seco } & \multicolumn{2}{|l|}{ Inundado } & \multicolumn{2}{|l|}{ Seco } & \multicolumn{2}{|c|}{ Inundado } & \multicolumn{2}{|l|}{ Seco } & \multicolumn{2}{|l|}{ Inundado } & \multicolumn{2}{|l|}{ Seco } & Inundado \\
\hline & \multicolumn{15}{|c|}{ Massa seca da parte aérea $(\mathrm{g})$} \\
\hline 0,00 & $22,48^{*} \quad \mathrm{~b}$ & $\mathrm{~b}^{2} /$ & $20,03 \quad \mathrm{a}$ & $\mathrm{a}$ & $21,30^{\mathrm{ns}}$ & $\mathrm{b}$ & 21,25 & $\mathrm{a}$ & $21,63^{\mathrm{ns}}$ & $\mathrm{b}$ & $21,55 \quad \mathrm{a}$ & & $24,15^{\mathrm{ns}}$ & & $19,95 \quad \mathrm{a}$ \\
\hline 3,125 & $6,13^{*} \mathrm{~b}$ & $\mathrm{~b}$ & $13,53 \mathrm{~b}$ & b & $19,98^{\mathrm{ns}}$ & $\mathrm{a}$ & 18,85 & a & $20,95^{\text {ns }}$ & a & $20,20 \quad \mathrm{a}$ & & $20,88^{\text {ns }}$ & & $20,10 \quad \mathrm{a}$ \\
\hline 6,25 & $1,60^{\text {ns }}$ a & $\mathrm{a}$ & $2,30 \quad \mathrm{a}$ & $\mathrm{a}$ & $1,78^{\mathrm{ns}}$ & a & 3,20 & $\mathrm{a}$ & $1,13^{\text {ns }}$ & $\mathrm{a}$ & $1,80 \quad \mathrm{a}$ & a & $0,88^{\text {ns }}$ & a & $2,08 \quad \mathrm{a}$ \\
\hline 12,50 & $1,88^{\text {ns }} \quad$ a & & $2,53 \quad \mathrm{a}$ & $\mathrm{a}$ & $1,65^{\text {ns }}$ & $\mathrm{a}$ & 2,50 & $\mathrm{a}$ & $1,18^{\text {ns }}$ & $\mathrm{a}$ & $1,55 \quad \mathrm{a}$ & $\mathrm{a}$ & $1,35^{\mathrm{ns}}$ & $\mathrm{a}$ & $1,95 \mathrm{a}$ \\
\hline 25,00 & $1,95^{\mathrm{ns}} \mathrm{a}$ & a & 1,93 a & a & $1,48^{\text {ns }}$ & $\mathrm{a}$ & $\begin{array}{l}1,68 \\
1,63\end{array}$ & a & $1,00^{\mathrm{ns}}$ & $a$ & 1,33 a & a & $1,08^{\text {ns }}$ & a & $1,50 \mathrm{a}$ \\
\hline 50,00 & $1,35^{\text {ns }} \quad$ a & $\mathrm{a}$ & $1,80 \quad \mathrm{a}$ & a & $1,33^{\mathrm{ns}}$ & a & 1,95 & $\mathrm{a}$ & $1,00^{\mathrm{ns}}$ & $\mathrm{a}$ & $1,33 \quad \mathrm{a}$ & a & $1,05^{\text {ns }}$ & a & $1,35 \quad \mathrm{a}$ \\
\hline \multirow[t]{2}{*}{100,00} & $1,33^{\text {ns }} \quad$ a & $\mathrm{a}$ & $1,70 \quad \mathrm{a}$ & $\mathrm{a}$ & $1,10^{\mathrm{ns}}$ & $\mathrm{a}$ & 1,40 & $\mathrm{a}$ & $1,18^{\text {ns }}$ & $\mathrm{a}$ & $1,20 \quad \mathrm{a}$ & $\mathrm{a}$ & $1,23^{\mathrm{ns}}$ & $\mathrm{a}$ & $1,33 \quad \mathrm{a}$ \\
\hline & \multicolumn{15}{|c|}{ Massa seca radical (g) } \\
\hline 0,00 & $39,28^{*} \quad \mathrm{a}$ & $\mathrm{a}$ & $27,40 \quad \mathrm{~b}$ & $\mathrm{~b}$ & $30,28^{*}$ & $b$ & 39,28 & $\mathrm{a}$ & $24,33^{\mathrm{ns}}$ & $\mathrm{c}$ & $24,68 \quad b$ & & $24,32^{\mathrm{ns}}$ & $\mathrm{c}$ & $27,22 \quad b$ \\
\hline 3,125 & $3,38^{*} \quad \mathrm{~d}$ & $\mathrm{~d}$ & $16,05 \mathrm{c}$ & $\mathrm{c}$ & $40,75^{\text {ns }}$ & a & 41,05 & $\mathrm{a}$ & $19,36^{*}$ & c & $26,45 \mathrm{~b}$ & & $29,48^{\text {ns }}$ & b & $29,10 \mathrm{~b}$ \\
\hline 6,25 & $0,68^{\mathrm{ns}} \quad \mathrm{a}$ & $\mathrm{a}$ & 1,68 a & a & $1,28^{\mathrm{ns}}$ & a & 2,83 & a & $1,30^{\mathrm{ns}}$ & a & $1,93 \quad \mathrm{a}$ & a & $0,73^{\mathrm{ns}}$ & a & $3,05 \quad \mathrm{a}$ \\
\hline 12,50 & $1,08^{\mathrm{ns}} \quad \mathrm{a}$ & $\mathrm{a}$ & $2,83 \quad \mathrm{a}$ & a & $0,90^{\text {ns }}$ & a & 1,53 & a & $0,68^{\mathrm{ns}}$ & a & $1,00 \quad \mathrm{a}$ & & $0,58^{\text {ns }}$ & $\mathrm{a}$ & $1,38 \quad \mathrm{a}$ \\
\hline 25,00 & $1,18^{\mathrm{ns}} \quad \mathrm{a}$ & $\mathrm{a}$ & 0,95 & a & $0,60^{\mathrm{ns}}$ & a & 0,53 & $\mathrm{a}$ & $0,40^{\text {ns }}$ & a & $0,70 \quad a$ & & $0,38^{\mathrm{ns}}$ & a & $0,53 \quad$ \\
\hline 50,00 & $0,65^{\text {ns }}$ & $\mathrm{a}$ & $0,85 \quad$ & $\mathrm{a}$ & $0,53^{\text {ns }}$ & a & 0,63 & a & $0,35^{\text {ns }}$ & $\mathrm{a}$ & $0,48 \quad \mathrm{a}$ & & $0,40^{\text {ns }}$ & a & $0,50 \quad \mathrm{a}$ \\
\hline 100,00 & $0,43^{\text {ns }} a$ & a & 0,40 a & a & $1,83^{\text {ns }}$ & $\mathrm{a}$ & 0,45 & a & $0,40^{\mathrm{ns}}$ & $\mathrm{a}$ & 0,33 a & $\mathrm{a}$ & $0,38^{\mathrm{ns}}$ & A & 0,40 a \\
\hline
\end{tabular}

${ }^{1 /}$ Dose de $100 \mathrm{~g} \mathrm{ha}^{-1}$ de imazethapyr + imazapic; ${ }^{2 /}$ Dias após a aplicação dos tratamentos. ${ }^{3 /}$ Médias acompanhadas por mesma letra na linha, comparando cultivares, em cada condição de aplicação, não diferem entre si pelo teste de Duncan a 5\% de probabilidade.

${ }^{n s}$ e * Não-significativo e significativo, respectivamente, pelo teste t a 5\% de probabilidade em função da condição de aplicação.

A resposta da fitotoxicidade do herbicida aos cultivares de arroz IRGA 417, BR-IRGA 410, BRS Pelota e Qualimax 1 ajustou-se à equação exponencial em todas as épocas de avaliação e condições de aplicação. Os valores do coeficiente de determinação $\left(\mathrm{R}^{2}\right)$ variaram de 0,82 a 0,97, demonstrando ajuste adequado dos dados ao modelo (Figuras 1 a 3).

Na primeira avaliação, para todos os cultivares estudados, à exceção de BR-IRGA 410, a fitotoxicidade foi superior quando a aplicação ocorreu em solo seco (Figura 1). A fitotoxicidade foi crescente com a dose de imazethapyr + imazapic em todos os cultivares estudados. Os valores máximos estimados pelo modelo, para aplicação no seco, foram em média 7,4\% superiores aos estimados para a aplicação em solo inundado. Resultado semelhante de fitotoxicidade foi observado no cultivar de arroz Qualimax 1, com o herbicida glyphosate, e EMBRAPA 6-Chuí, para butroxydim, os quais aumentaram linearmente aos 10 DAT, em deriva simulada sob condições de solo seco (Ferreira, 2003).

Na segunda avaliação, os sintomas de fitotoxicidade aumentaram em todos os cultivares e nas duas condições de aplicação testadas
(Figura 2). Nesta avaliação, o cultivar BRS Pelota apresentou maior fitotoxicidade nas duas condições de aplicação. Esses resultados assemelham-se aos verificados no cultivar de arroz Cypress, no qual as injúrias causadas por deriva de glyphosate atingiram 98\% aos 14 DAT, demonstrando que a sensibilidade pode variar em função do herbicida utilizado (Ellis et al., 2003).

Aos 20 DAT, a fitotoxicidade aumentou em todos os cultivares (Figura 3). As plantas de arroz apresentaram injúrias que atingiram valores médios de 95,8\% quando a aplicação ocorreu em lavoura no seco e de 93,8\% quando ela ocorreu em lavoura inundada. Para o herbicida glyphosate, aplicado no estádio de crescimento V3 - V4, injúrias de 65 e 50\% foram observadas nos cultivares Lemont e Priscilla, respectivamente (Kurtz \& Street, 2003).

Os maiores valores do parâmetro b, observados no cultivar IRGA 417 em todas as épocas de avaliação e condições de aplicação, demonstraram que esse cultivar é mais sensivel aos efeitos fitotóxicos da deriva simulada do herbicida imazethapyr + imazapic. Esses resultados corroboram os observados na Tabela 1 . 

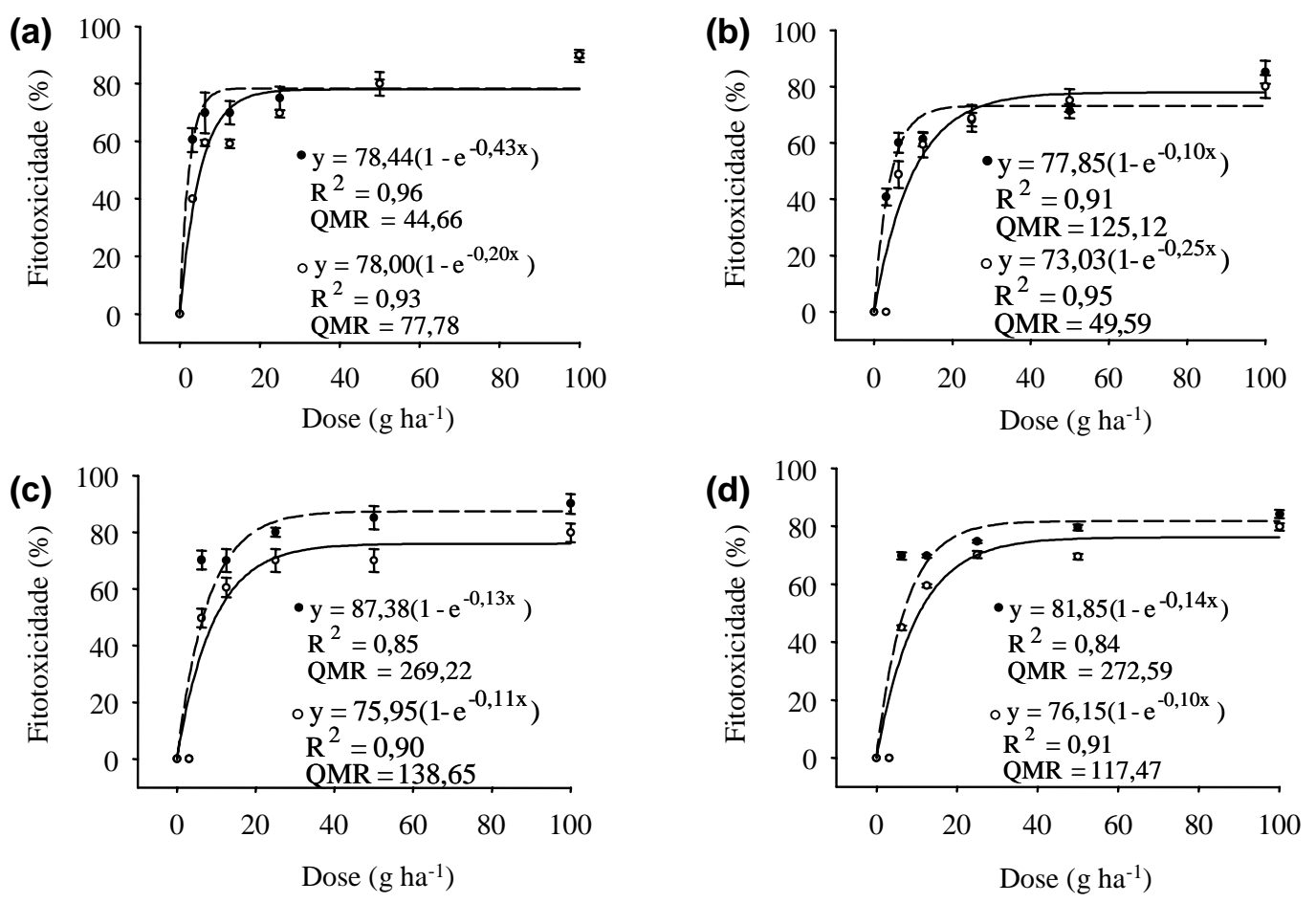

Figura 1 - Fitotoxicidade de imazethapyr + imazapic para a cultura do arroz irrigado, cultivares IRGA 417 (A), BR-IRGA 410 (B), BRS Pelota (C) e Qualimax 1 (D), em função de dose e condição de aplicação, aos 10 dias após a aplicação dos tratamentos (DAT). CAP/UFPel, Capão do Leão-RS, 2003/04. (•: aplicação em solo seco; O: aplicação em solo inundado).
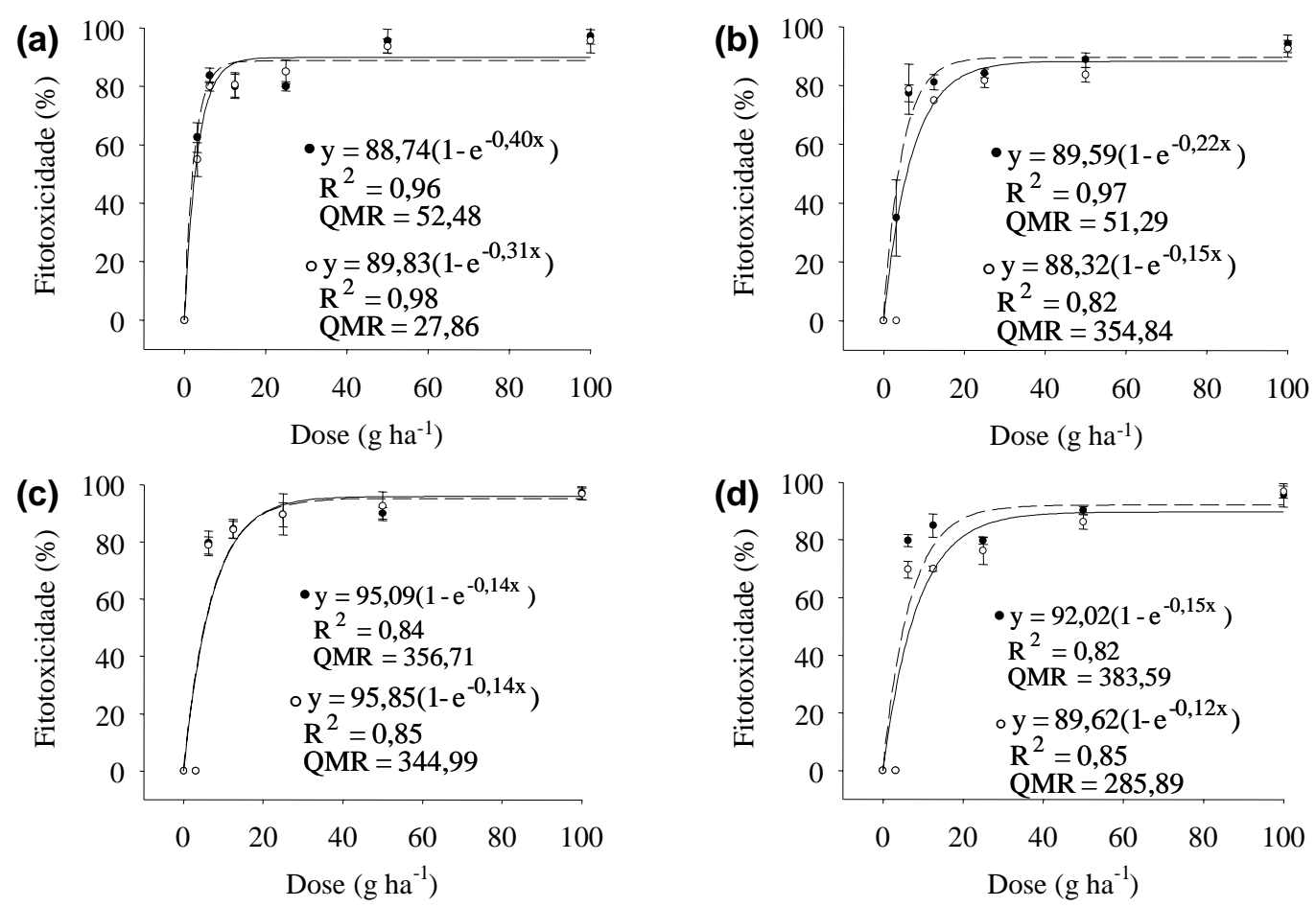

Figura 2 - Fitotoxicidade de imazethapyr + imazapic para a cultura do arroz irrigado, cultivares IRGA 417 (A), BR-IRGA 410 (B), BRS Pelota (C) e Qualimax 1 (D), em função de dose e condição de aplicação, aos 15 dias após a aplicação dos tratamentos (DAT). CAP/UFPel, Capão do Leão-RS, 2003/04. (•: aplicação em solo seco; O: aplicação em solo inundado). 

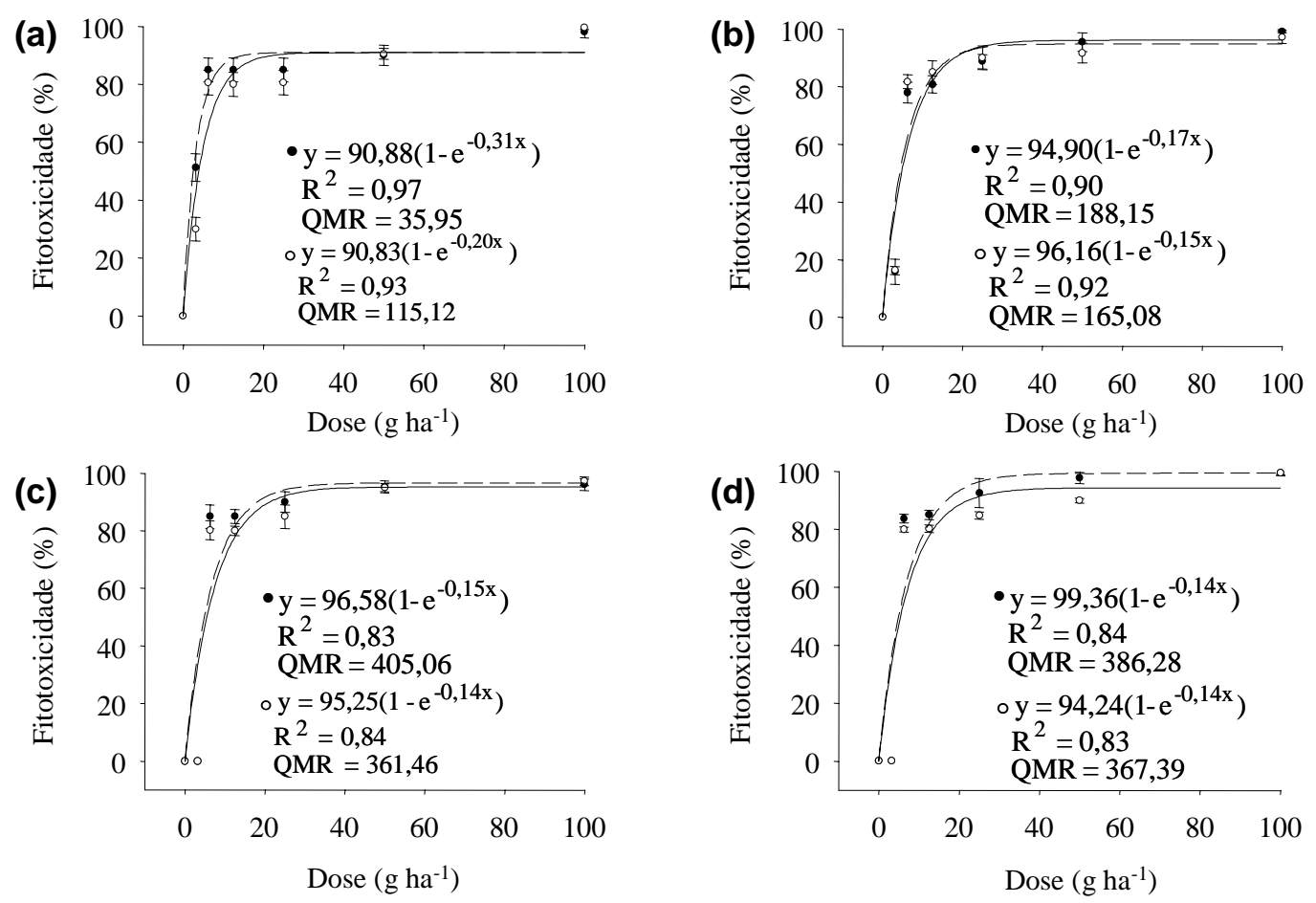

Figura 3 - Fitotoxicidade de imazethapyr + imazapic para a cultura do arroz irrigado, cultivares IRGA 417 (A), BR-IRGA 410 (B), BRS Pelota (C) e Qualimax 1 (D), em função de dose e condição de aplicação, aos 20 dias após a aplicação dos tratamentos (DAT). CAP/UFPel, Capão do Leão-RS, 2003/04. (•: aplicação em solo seco; O: aplicação em solo inundado).

A produção de massa seca da parte aérea e radical dos cultivares de arroz ajustou-se à equação exponencial em todas as condições de aplicação (Figuras 4 e 5). Os valores do coeficiente de determinação $\left(\mathrm{R}^{2}\right)$ variaram de 0,78 a 0,96, apresentando bom ajuste dos dados observados ao modelo.

A produção de massa seca da parte aérea foi reduzida com o aumento da dose em todos os cultivares testados e condições de aplicação (Figura 4). Resultados semelhantes, em campo, foram observados por Ferreira (2003), para os cultivares Qualimax 1 e EMBRAPA 6-Chuí, em que a deriva simulada de glyphosate e butroxydim, respectivamente, reduziu a produção de matéria seca.

A produção de massa seca radical foi reduzida com o aumento da dose de imazethapyr + imazapic, para todos os cultivares testados e condições de aplicação, da mesma forma que o verificado para a variável massa seca da parte aérea (Figura 5).

A inclinação da curva, parâmetro b, foi superior no cultivar IRGA 417 nas duas condições de aplicação, para as variáveis massa seca da parte aérea e radical (Figuras 4 e 5). Esses resultados demonstram que o cultivar IRGA 417 é mais sensivel aos efeitos fitotóxicos de deriva de imazethapyr + imazapic e corroboram os apresentados na Tabela 2 , em que a menor produção de massa seca da parte aérea e radical também foi observada no cultivar IRGA 417.

Os resultados demonstram haver diferença de suscetibilidade entre cultivares quanto ao herbicida imazethapyr + imazapic em função da ocorrência de deriva. Neste estudo, o cultivar que se mostrou mais sensivel e tolerante à deriva simulada de imazethapyr + imazapic foi o IRGA 417 e BR-IRGA 410, respectivamente, independentemente da condição de aplicação. A elevada fitotoxicidade e baixa produção de massa seca aérea e radical observada nas menores doses herbicidas podem decorrer da pequena quantidade de solo presente nos baldes, não permitindo que o herbicida seja dissipado, ou das condições climáticas internas da casa de vegetação, as quais podem interferir na atividade do herbicida na planta. 

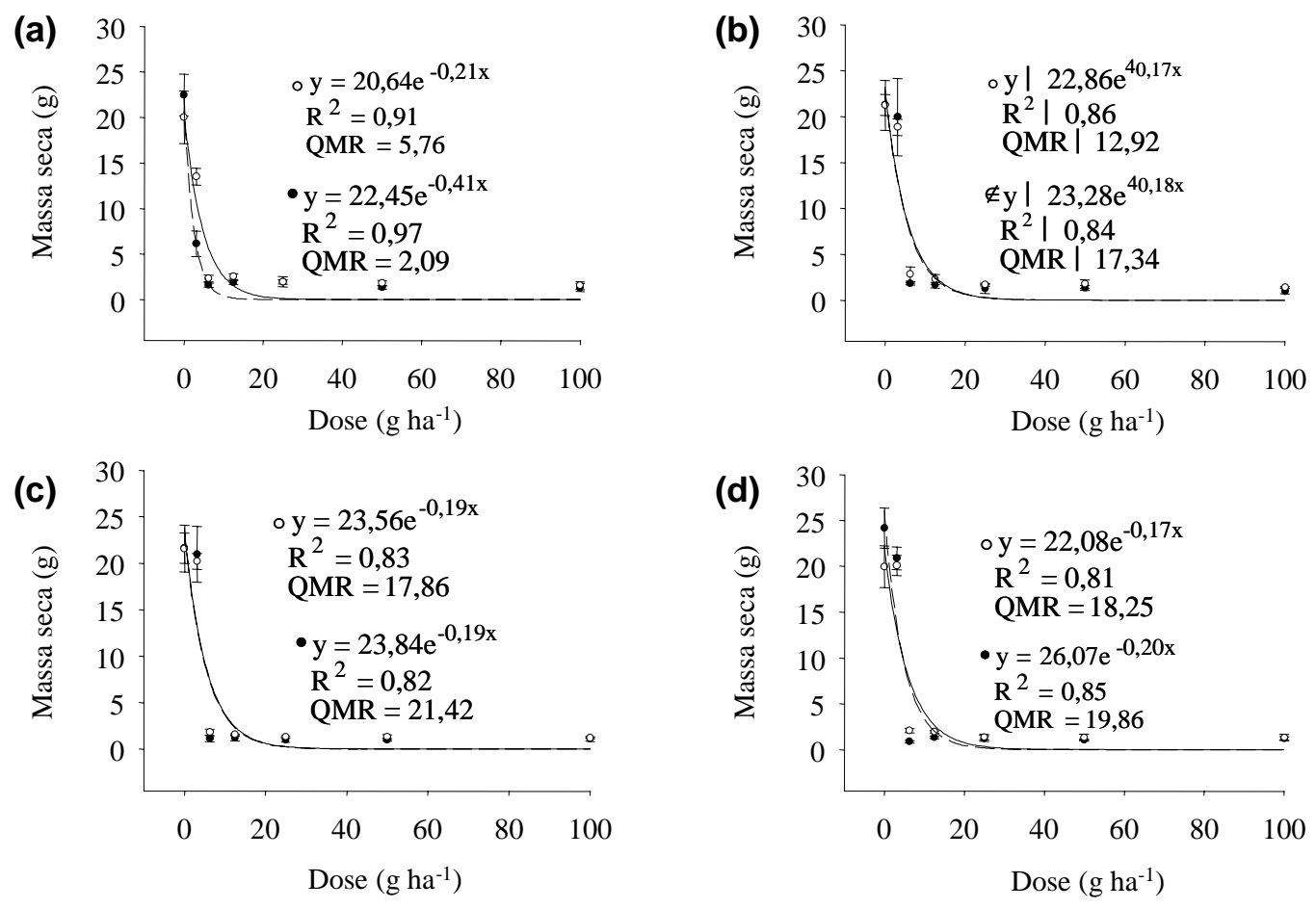

Figura 4 - Massa seca da parte aérea de plantas de arroz irrigado, cultivares IRGA 417 (A), BR-IRGA 410 (B), BRS Pelota (C) e Qualimax 1 (D), em função de dose e condição de aplicação aos 25 dias após a aplicação dos tratamentos (DAT). CAP/UFPel, Capão do Leão-RS, 2003/04. (•: aplicação em solo seco; O: aplicação em solo inundado).
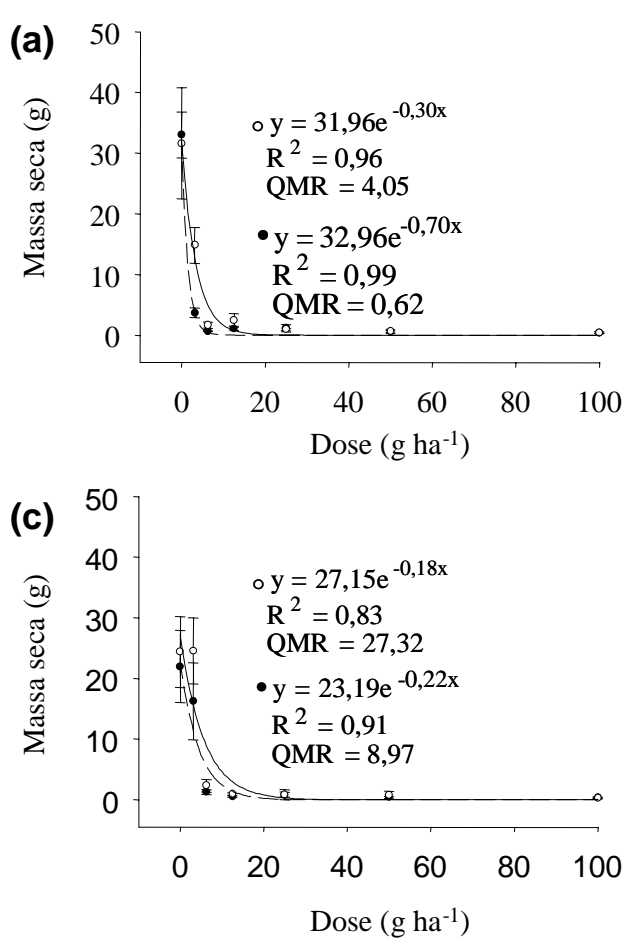
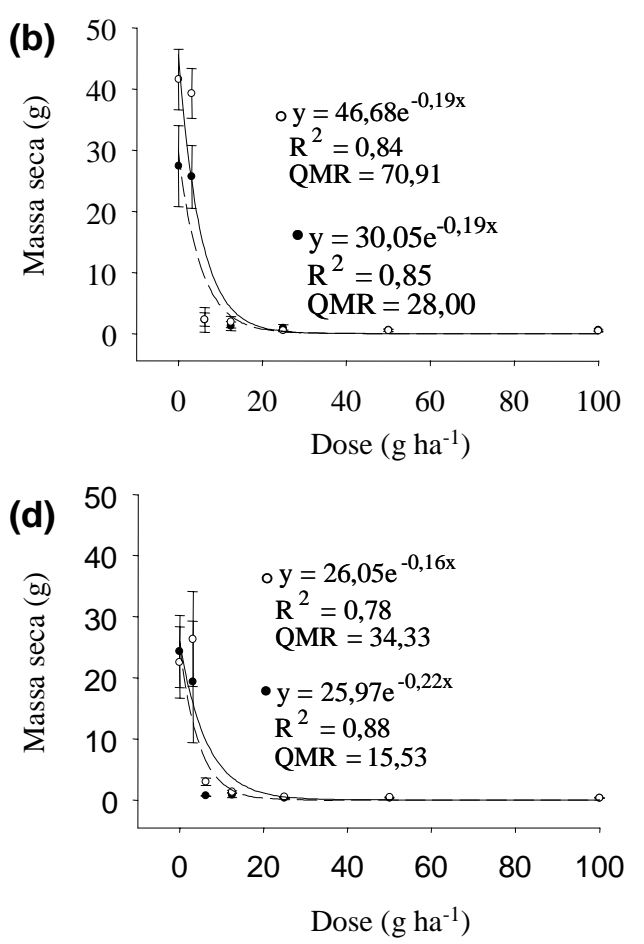

Figura 5 - Massa seca radical de plantas de arroz irrigado, cultivares IRGA 417 (A), BR-IRGA 410 (B), BRS Pelota (C) e Qualimax 1 (D), em função de dose e condição de aplicação, aos 25 dias após a aplicação dos tratamentos (DAT). CAP/UFPel, Capão do Leão-RS, 2003/04. (•: aplicação em solo seco; O: aplicação em solo inundado). 
O herbicida imazethapyr + imazapic, em deriva simulada, causa fitotoxicidade aos cultivares de arroz IRGA 417, BR-IRGA 410, BRS Pelota e Qualimax 1, quando ocorre em solo seco ou inundado. A massa seca da parte aérea e radical é reduzida com o aumento da dose aplicada, independentemente da condição de aplicação. Os cultivares IRGA 417 e BR-IRGA 410 foram os mais sensiveis e tolerantes, respectivamente, à deriva simulada de imazethapyr + imazapic.

\section{LITERATURA CITADA}

BENITEZ, C. A. G. Tolerância de cultivares de arroz irrigado a herbicidas. 1997. 48 f. Dissertação (Mestrado em Fitossanidade) - Universidade Federal de Pelotas, Pelotas, 1997.

ELEFTHEROHORINOS, I. L.; DHIMA, K. V. Red rice (Oryza sativa) control in rice (O. Sativa) with preemergence and postemergence herbicides. Weed Technol., v. 16, p. 537540, 2002.

ELLIS, J. M. et al. Rice (Oryza sativa) and corn (Zea mays) response to simulated drift of glyphosate and glufosinate. Weed Technol., v. 17, p. 452-460, 2003.

EMPRESA BRASILEIRA DE PESQUISA AGROPECUÁRIA - EMBRAPA. Centro Nacional de Pesquisa de Solos. Sistema brasileiro de classificação de solos. Rio de Janeiro: 1999. 412 p.

FERREIRA, F. B. Resposta do arroz irrigado (Oryza sativa) a subdoses de glyphosate e butroxydim simulando efeito de deriva. 2003. 52 f. Dissertação (Mestrado em Fitossanidade) - Universidade Federal de Pelotas, Pelotas, 2003.
FERRERO, A. et al. Mechanical and chemical control of red rice (Oryza sativa L. var. 'sylvatica') in rice (Oryza sativa) pre-planting. Crop Protec., v. 8, p. 45-251, 1999.

KURTZ, M.; STREET, J. E. Response of rice (Oryza sativa) to glyphosate applied to simulate drift. Weed Technol., v. 17, p. 234-238, 2003.

NOLDIN, J. A. et al. Desempenho de populações híbridas F2 de arroz-vermelho (Oryza sativa) com arroz transgênico (O. sativa) resistente ao herbicida amonio-glufosinate. Planta Daninha, v. 22, n. 3, p. 381-395, 2004.

OTTIS, B. V.; CHANDLER, J. M; McCAULEY, G. M. Imazethapyr application methods and sequences for imidazolinone-tolerant rice (Oryza sativa). Weed Technol., v. 17 , p. 526-533, 2003.

PINTO, J. J. O. et al. Controle de arroz daninho através do sistema de manejo Clearfield ${ }^{\circledR}$. In: CONGRESSO BRASILEIRO DE ARROZ IRRIGADO, 3., 2003, Balneário de Camboriú. REUNIÃO DA CULTURA DO ARROZ IRRIGADO, 25., 2003, Balneário de Camboriú. Anais... Balneário de Camboriú-SC: 2003. p. 468-470.

SIGMAPLOT - Exact Graphy for Exact Science. Version 8.0, 1999.

SOCIEDADE SUL-BRASILEIRA DE ARROZ IRRIGADO - SOSBAI. Arroz irrigado: recomendações técnicas da pesquisa para o Sul do Brasil. Itajaí: 2003. $126 \mathrm{p}$.

ZONTA, E. P.; MACHADO, A. A.; SILVEIRA JÚNIOR, P. Sistema de análise estatística para microcomputadores SANEST (software). Pelotas: Universidade Federal de Pelotas, 1984 